\title{
Research Paper \\ Changes in the Expression of SGO1 and SGO1-AS1 Genes in Colorectal Tumor Tissues, Compared to Healthy Tissues
}

\author{
Mojtaba Asad Samani ${ }^{1}$ 이, ${ }^{*}$ Maryam Peymani ${ }^{1}$ 이
}

1. Department of Biology, Faculty of Basic Sciences, Shahrekord Branch, Islamic Azad University, Shahrekord, Iran.



Citation: Samani AM, Peymani M. [Changes in the Expression of SGO1 and SGO1-AS1 Genes in Colorectal Tumor Tissues, Compared to Healthy Tissues (Persian)]. Journal of Arak University of Medical Sciences (JAMS). 2021; 24(2):168-179. https:// doi.org/10.32598/JAMS.24.2.5136.1

doi hittps://doi.org/10.32598/JAMS.24.2.5136.1

Key words: SGO1 gene, SGO1 -AS1 gene, Colorectal cancer, ROC curve

\section{A B STRACT}

Article Info:

Received: 15 Jul 2020

Accepted: 25 Aug 2020

Available Online: 01 Jun 2021
Background and Aim The protein encoded by the SGO1 gene is a member of the shugoshin family of proteins and protects the centromere during mitosis. IncRNAs are non-coding RNA with 200 nucleotides lengths, i.e., involved in regulating gene expression. The current study aimed to evaluate the expression of SGO1 and SGO1-AS1 in different stages of disease progression; we also compared their expression pattern in tumor tissues with healthy tissues in colorectal cancer patients.

Methods \& Materials In total, 40 tissue samples of patients with colorectal cancer were reported according to the examination and criteria with the approval of a pathologist. Besides, 40 normal tissues were sampled from a completely healthy part of the intestine of the same patients. After RNA extraction and cDNA synthesis, the Real-time RT-PCR technique was used to evaluate the expression of the desired genes in the study groups. ROC curve analysis was also used to determine the ability of each selected gene to diagnose the disease.

Ethical Considerations This study was approved by the Ethics Committee of Shahrekord Azad University (Code: IR.IAU.SHKREC.1398.020).

Results The obtained data suggested that SGO1 significantly decreased in the colorectal cancer tumor samples $(\mathrm{P}<0.001)$ and SGO1-AS1 LncRNA significantly increased expression, compared to adjacent healthy tissues. Additionally, in the age group of below 60 years, compared to the age group of over 60 years, SGO1 expression increased and SGO1-AS1 expression decreased. Based on the AUC obtained from the ROC diagram, it was found that the SGO1 gene with $A U C=0.8041$ and SGO1-AS1 with AUC $=0.6364$ could significantly distinguish a healthy population from patients with colorectal cancer.

Conclusion According to the collected results, SGO1 -AS1 and SGO1 were significantly reduced and increased in tumor tissue, respectively; however, only the $S G O 1$ gene was introduced as a good marker for diagnosing colorectal cancer.

\section{Extended Abstract}

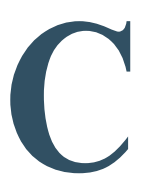

\section{Introduction}

olorectal cancer is among the leading causes of death and is the fourth most common cancer worldwide [1] that ranks third among women [2-5]. The $S G O 1$ protein is a member of the shugoshin protein family. Besides, and decreased expression of the SGO1 gene leads to the premature destruction of the centromere during mitosis [6-8]. Mutations in the SGO1 gene lead to transcriptomic changes in metabolism, proliferation, and immune responses in the gut that contribute to cancer progression [9]. IncRNAs play an important

\section{* Corresponding Author:}

Maryam Peimani, Msc.

Address: Department of Biology, Science Faculty, Shahrekord Branch, Islamic Azad University, Shahrekord, Iran.

Tel: +98 (913) 2007650

E-mail:m.peymani@iaushk.ac.ir 
role in controlling cell growth by regulating the cell cycle and apoptosis [10]. Growth Arrest-Specific (GAS5) accumulates in stunted growth cells and sensitizes mammalian cells to apoptosis [11]. Regarding the role of SGO1-AS1 and subsequent tumorigenesis and regulation of $S G O 1$ gene expression, in this study, for the first time, the expression of SGO1-AS1 and SGO1 genes in colorectal cancer tumor tissue was compared with healthy tissue.

\section{Materials and Methods}

The present case-control study was performed on 40 tumor tissues of 40 individuals with colorectal cancer and 40 adjacent healthy tissues. Trizol was used to extract total RNA and after qualitative and quantitative analysis, the cDNA of each sample was synthesized using the kit of Yekta Tajhiz Azma Company. Using the Real-Time-RT PCR technique and especially, designed reciprocating primers, a quantitative measurement of the expression level of the desired genes was performed. In this study, after obtaining the relative frequency of expression for $S G O 1$ and $S G O 1-A S 1$ genes in colorectal cancer, different tests were implemented to compare the obtained data.

GraphPad Prism and Excel software were used to analyze the collected data; after confirming the normality of the sample size with the Shapiro test, a t-test was used to examine the difference in the expression of $S G O 1 \mathrm{~S}$ and SGO1-AS1 genes in tumorous and healthy samples. OneWay Analysis of Variance (ANOVA) was used to compare

A

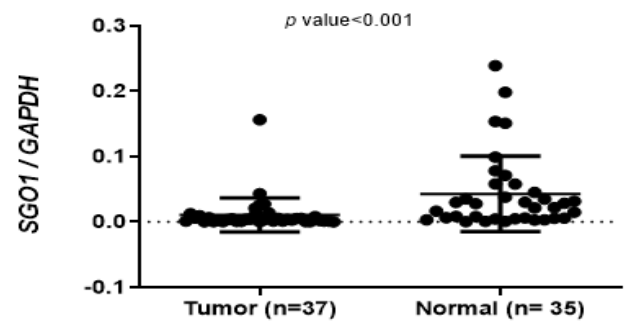

C

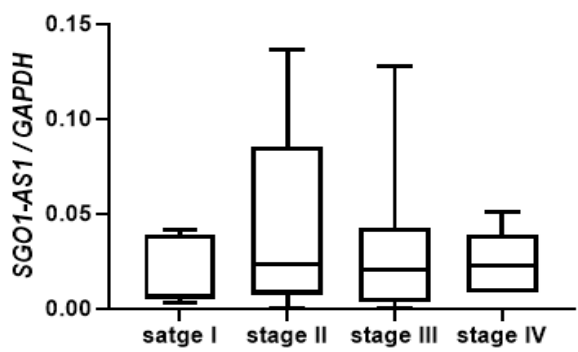

the expression of genes in different stages. Spearman test was also used to examine the correlation of expression of the desired genes. Finally, to evaluate the specificity and sensitivity of each gene, the ROC test was applied to plot the ROC Curve.

\section{Results}

As shown in Figure 1A, the expression level of SGO1 was significantly reduced in tumor samples, compared to healthy tissue $(\mathrm{P}<0.001)$. However, the expression level of $S G O 1-A S 1$ in tumor tissue presented a significant increase, compared to the healthy tissue (Figure 1B) $(\mathrm{P}=0.0116)$. The expression levels of $S G O 1$ and SGO1-AS1 in different stages of the disease were analyzed in tumor tissues. The relevant results indicated that the expression level of these genes remained unchanged at different stages of the disease (Figures 1C \& D).

The expression level of SGO1 in the age group under 60 years illustrated less expression; however, the expression level of SGO1-AS1 revealed a significant increase in this age group, compared to the age group over 60 years. Figures $2 \mathrm{~A}$ and $\mathrm{B}$ demonstrate a graph of changes in the relative expression levels of genes at the $\mathrm{Ct} \Delta-2$ level in both age groups in tumor tissues.

The results concerning the ROC curve diagram indicated that the marker SGO1-AS1 with the area below the surface of the diagram ( $\mathrm{AUC}=0.6364 \& \mathrm{CI}=0.5069 / 7669)$ acted as a poor marker in the diagnosis of colorectal can-

\section{B}

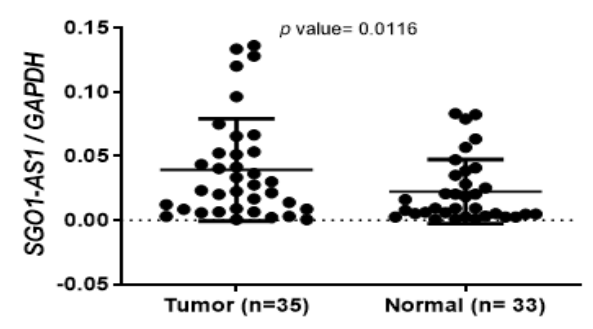

D

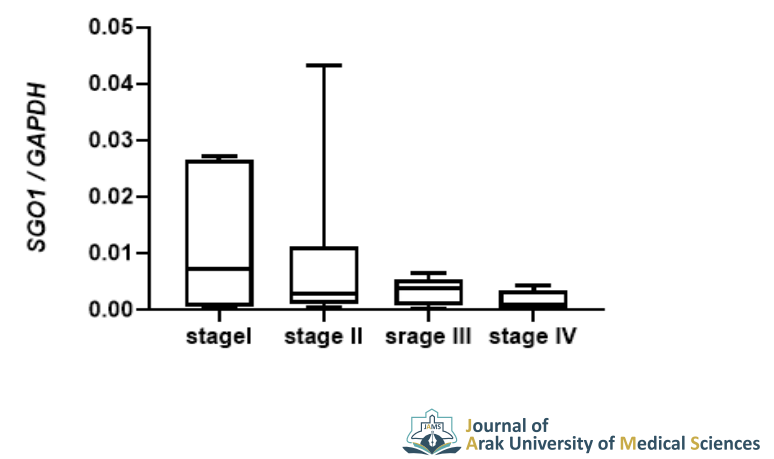

Figure 1. The changes in the relative expression levels of $S G O 1-A S 1$ and $S G O 1$ in tumor and healthy tissues 



Figure 2. SGO1 expression levels in the age groups of under and over 60 years

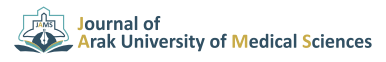

cer (Figure 3A). However, SGO1, as a marker can significantly $(\mathrm{P}<0.0001)$ separate the patient population from the healthy groups; with the area below the surface of the chart (AUC $=0.8041 \& \mathrm{CI}=0.7036 / 9045)$, it can be a good marker to help improve the diagnosis of colorectal cancer (Figure 3B).

\section{Discussion and Conclusion}

The present study data indicated that in the tumor tissues of colorectal cancer, the expression of the $S G O 1$ gene decreases, and the expression of the SGO1-ASI gene increases, compared to healthy tissue. In other words, the $S G O 1$ gene acts as a tumor suppressor and the SGO1-AS1 gene as an oncogene. In a 2006 study of the SGO1 gene, Yang et al. Stated that human SGO1 has become a good target for inducing apoptosis into transformed cells [15]. In 2015,

A

ROC curve: ROC of SGO1-AS1



Wang et al. examined the SGO1 gene in liver cancer. They stated that the SGO1 gene is a potential therapeutic target for liver cancer [19]. In 2018, Ong et al. reported that an increase in lncRNA expression is detected in individuals with colorectal cancer [20].

In 2019, Mu et al. argued that SGO1 expression levels were higher in PCA (prostate cancer) tissue and cell lines. There was a correlation between SGO1 expression and preoperative prostate-specific antigen $(\mathrm{P}=0.01)$. Furthermore, gene expression was significantly associated with lymph node metastasis $(\mathrm{P}=0.044)$ [21]. This study also revealed that $S G O 1$ decreased expression in colorectal cancer tumor samples. Measuring SGO1-AS1 expression in tumor and healthy colorectal cancer samples also identified an increase in LncRNA expression; these two genes could be used as a marker for the diagnosis of colorectal cancer.

B

ROC curve: ROC of SGO1



Figure 3. The specificity and sensitivity of $S G O 1$ and $S G O 1-A S 1$ in colorectal cancer 


\section{Ethical Considerations}

Compliance with ethical guidelines

This study was approved by the Ethics Committee of Shahrekord Azad University (Code: IR.IAU. SHKREC.1398.020).

\section{Funding}

This study was extracted from the MSc. thesis of the first author at the Department of Biology, Faculty of Basic Sciences, Shahrekord Branch, Islamic Azad University, Shahrekord, Iran. Also, this study was supported by the Vice Chancellor for Research of Islamic Azad University, Shahrekord.

\section{Authors' contributions}

Both authors met standard writing standards based on recommendations from the International Committee of Medical Journal Publishers.

\section{Conflicts of interest}

The authors declared no conflicts of interest. 
This Page Intentionally Left Blank 
تغييرات در بيان ثنهاي SGO1 و SGO1-AS1 در بافتهاي تومورى كلوركتال در مقايسه با بافتهاى سالم

\author{
مجتبى اسعد سامانى' هـ، "مريم ييمانى'
}

1. كروه زيست شناسي، دانشكده علوم هايه، واحد شهركرد، دانشكاه آزاد اسلامى، شهركرد، ايران.

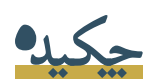





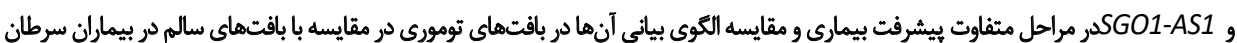
كلوركثال است.





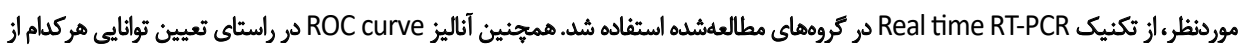



هلاحظات اخلاقي، ائ يُروهش در كميته اخلاق مانشكاه آزاد شهركرد با كد

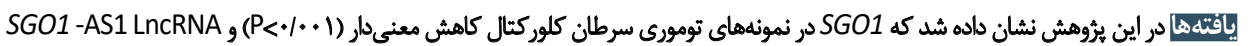

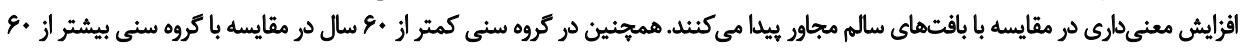

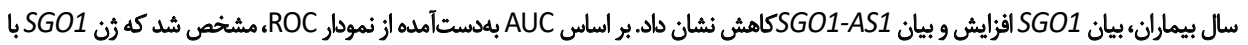
AUC=./9Y94 به طور معنى دارى قابليت تشخيص جمعيت سالم از بيمار رادر سرطان كلوركتال دارند.

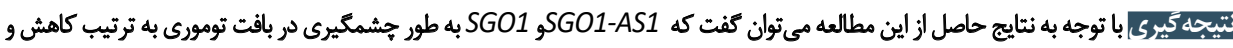

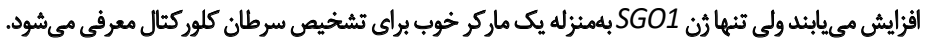





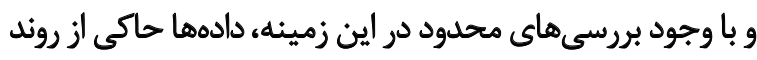



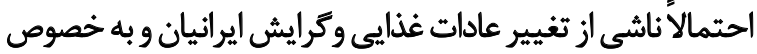

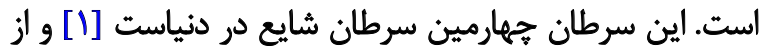



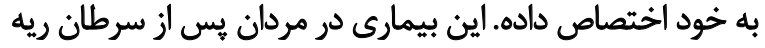

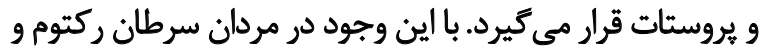



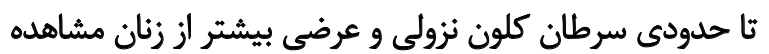


آيويتوز مرك برنامهريزي شده سلول است كه عاملهاي متعددى

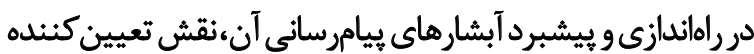


سلولى و آيويتوز نقش مهيمى در كنترل رشد سلول ايفا مي كنئن

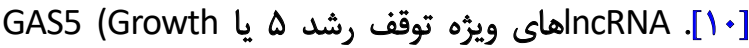
arrest-specific)

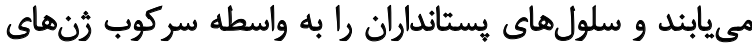

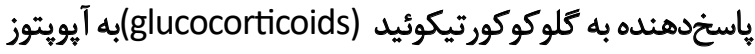
حساس مىسازد. GAS5 با كيرنده كلوكوكئيد

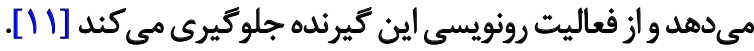

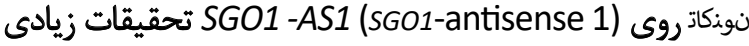

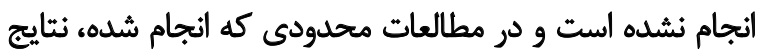

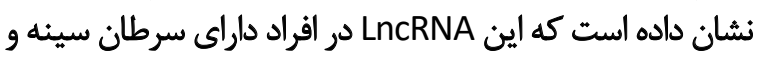







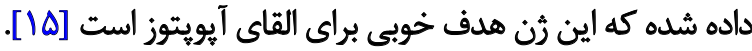

shugoshin عضوخانوادهيروتئينين

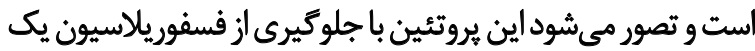







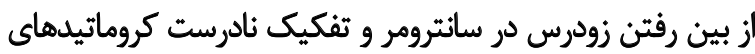

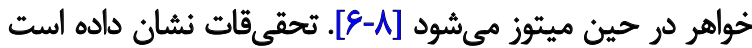

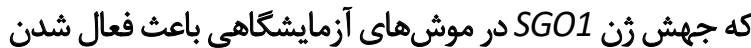

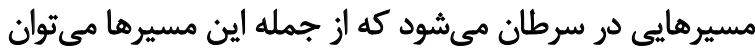

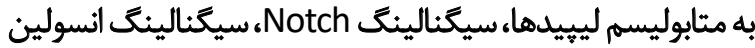

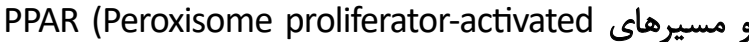

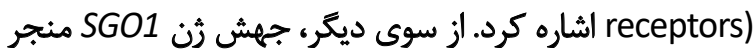

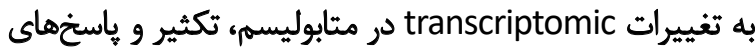

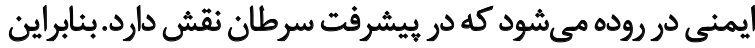





[ز رشمئد باشد [9].

جدول ا. متغيرهاى جمعيتشُناختى و اطلاعات بالينى




جدول r. آغازكرهاى استفادهشده، آغاز كرهاى GAPDH , SGO1-AS1 SGO1 به همراه طول محصول و دماى آنها در روش RT-qPCR

\begin{tabular}{|c|c|c|c|}
\hline Ta $\left({ }^{\circ} \mathrm{C}\right)$ دماى & طول محصول (bp) & توالى آغازتر & ن ام آغازتر \\
\hline q. & 1.4 & $\begin{array}{l}\text { Reverse: } \Delta^{\prime}-\text { - CTCCTTGTCCTGGAAGTTCAGT -r' } \\
\text { Forward: } \Delta^{\prime} \text { - TCCTCTGGAATGGACCCCAA -r' }\end{array}$ & $S G O 1$ \\
\hline q. & iva & $\begin{array}{c}\text { Reverse: } \Delta^{\prime} \text { - GGAGTCCCTAAATCTGTTTAATGTC -r' } \\
\text { Forward: } \Delta^{\prime} \text { - GCTCACTTTTGTCGGAAGG -r' }\end{array}$ & SG01-AS1 \\
\hline s. & MI & $\begin{array}{l}\text { Reverse: } \Delta^{\prime} \text {-TGAAGTCGCAGGAGACAACC-r'" } \\
\text { Forward: ه' -TGCCGCCTGGAGAAACC-"r' }\end{array}$ & GAPDH \\
\hline
\end{tabular}

دقيقه در دماى ه \& درجه سانتى كراد انكوبه شد. سيس با استفاده

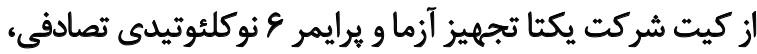

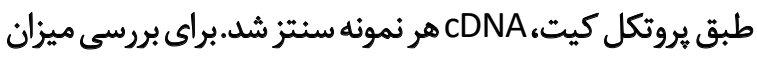

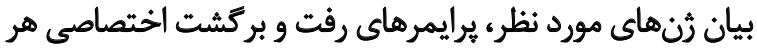

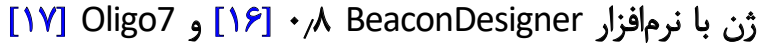

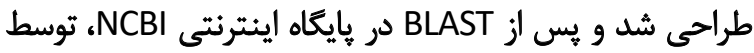

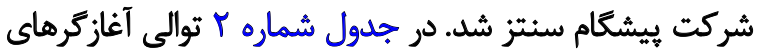

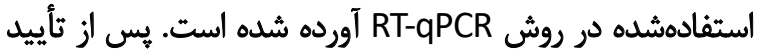
صحت سنتز CDNA، از تكنيك Real Time-RT PCR (دستكاه (9... Corbett rotor gene SYBRR

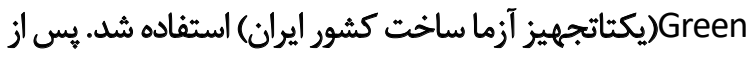

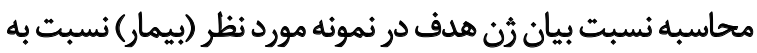

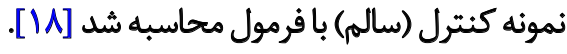

\section{s)}

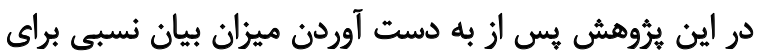

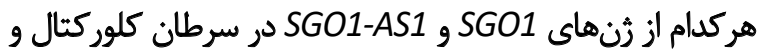

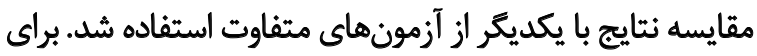

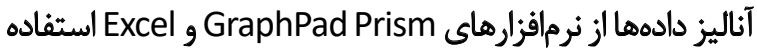
شد و يس از تأييد نرمال بودن حجم نمونه با آزمون Shapiro

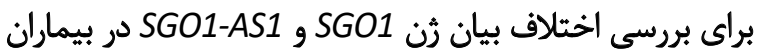

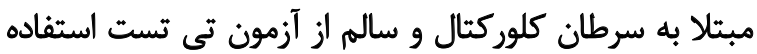

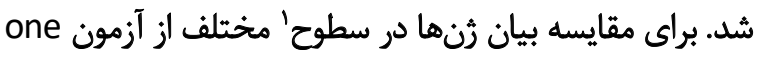
way ANOVA

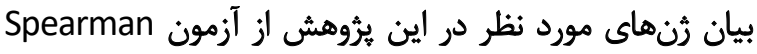

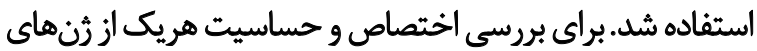

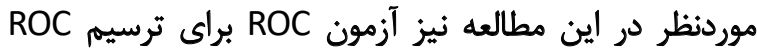
Curve

1. Stages
با توجه به نقش SGO1-AS1 در كلوكوكورتيكوئيد و به دنبال آن

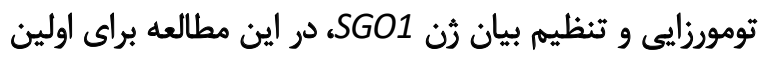

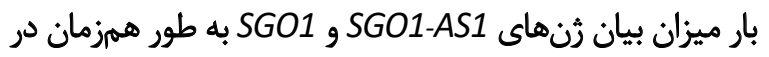
بافت تومورى سرطان كلوركتال در مقايسه با بافت سالم بررسى ونمان

شُ بـ.

مواد ورش روشا

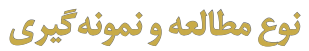

تحقيق حاضر در كميته اخلاق دانشعاه آزاد شهركرد با كد

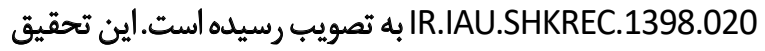

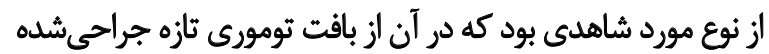

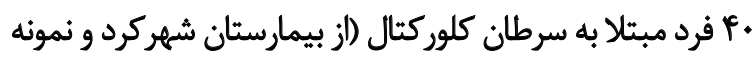

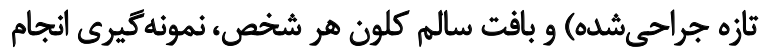

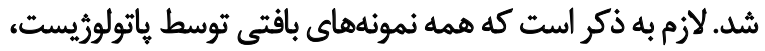

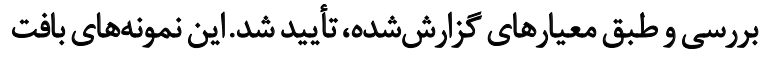

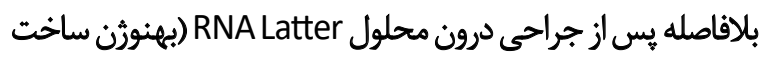

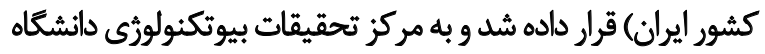

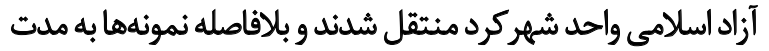

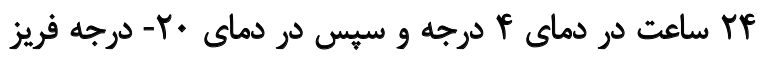

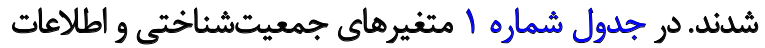

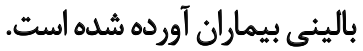

\section{Real time RT-PCR, cDNA سنّز}

براى استخراج RNA تام از ترايزول (nvitrogen ساخت كشور

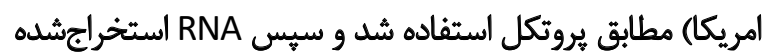

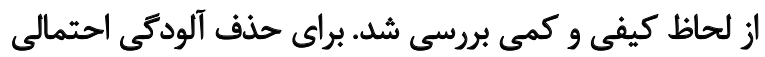

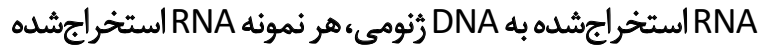

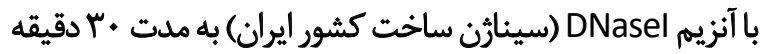

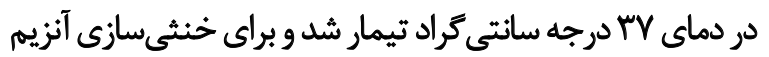

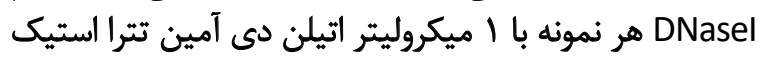

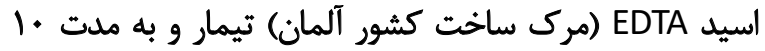




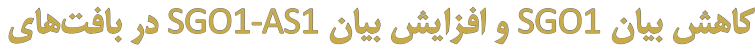

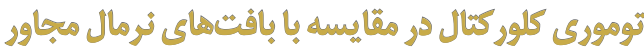

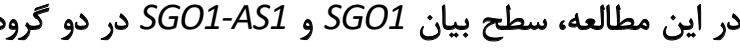

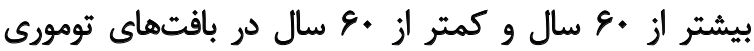

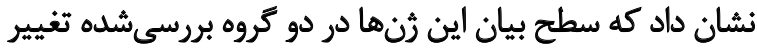

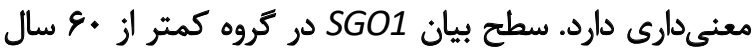

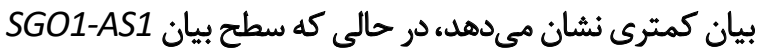

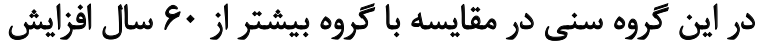

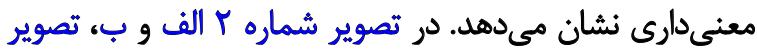

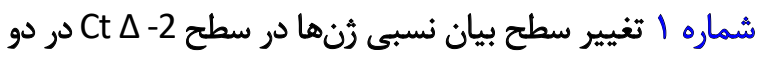
كروه سنى در بافتهاى تومورى نشان داده شده است

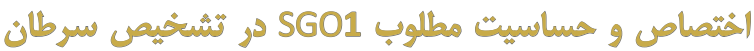
كالور كثأل

Sتشايج بر اساس نمولار ROC curve نشان داد كه ماركر

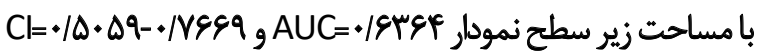

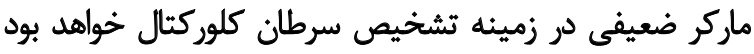

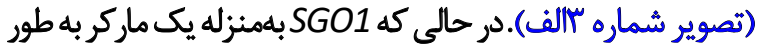

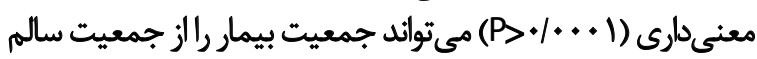

همان طور كه در تصوير شماره ا الف مشاهده مىشود، سطح بيان

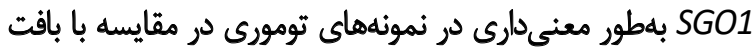

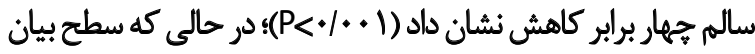

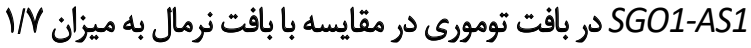

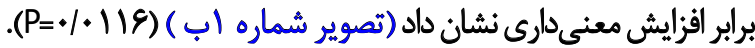

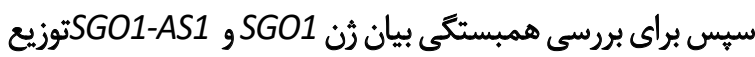

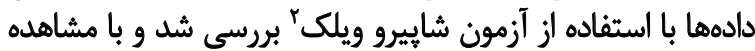

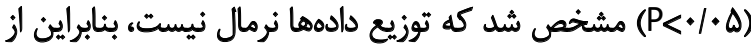

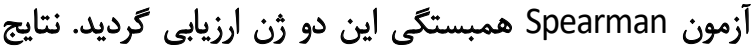

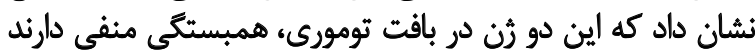

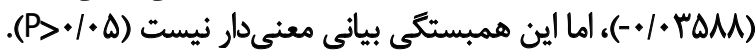

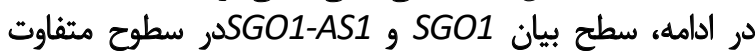
بيمارى در بافتهاى تومورى آناليز شد. نتايج نشان داد كاد كه سطح مئح

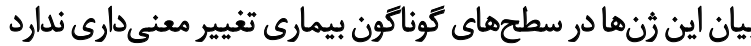

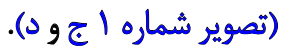

2. Shapiro Wilk

A

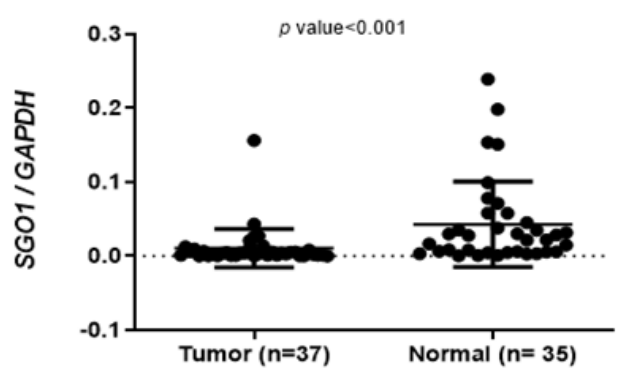

C

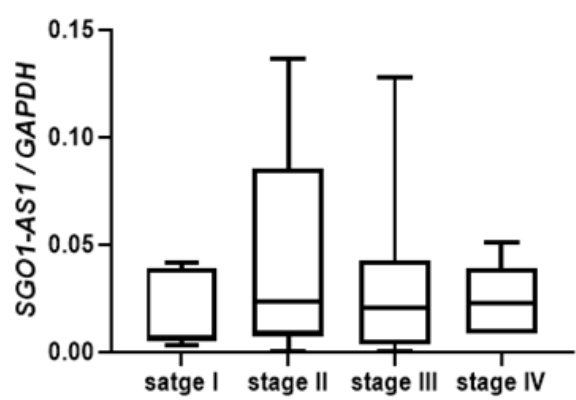

B

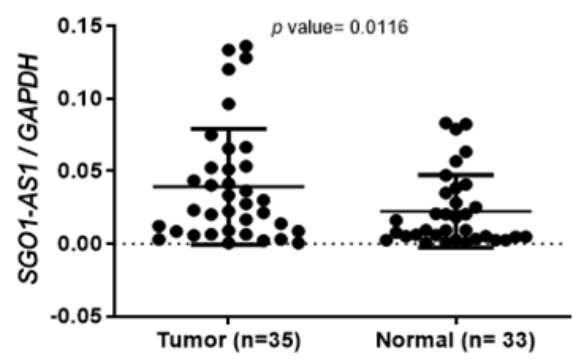

D

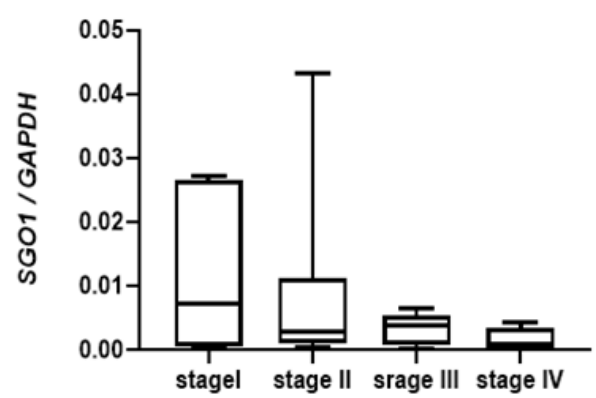

تصوير ا. بيان SGO1 و افزايش بيان SGO1-AS1 در بافتهاى تومورى كلوركتال در مقايسه با بافتهاى نرمال مجاور

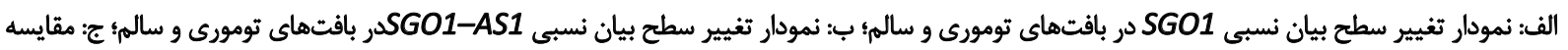

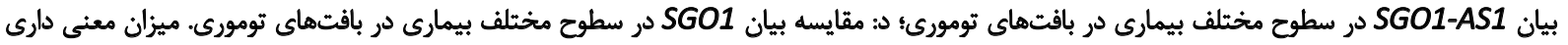

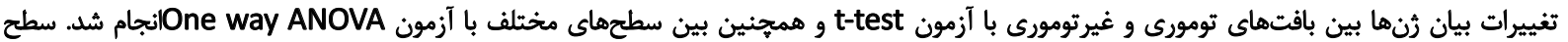


A

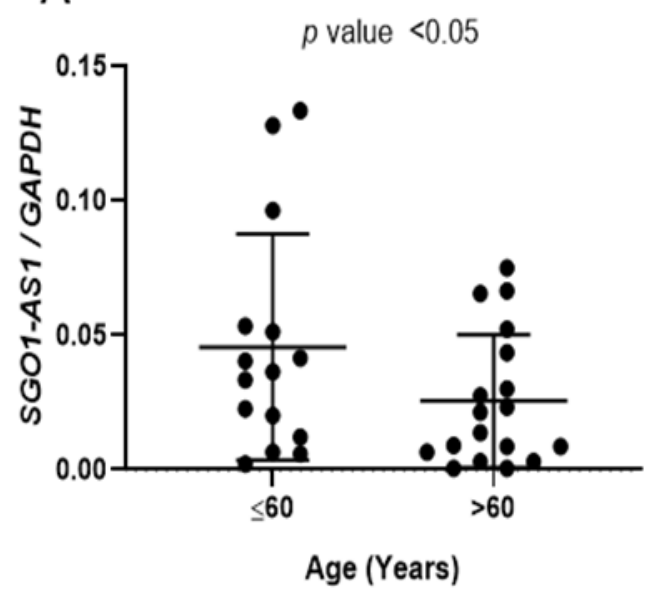

B

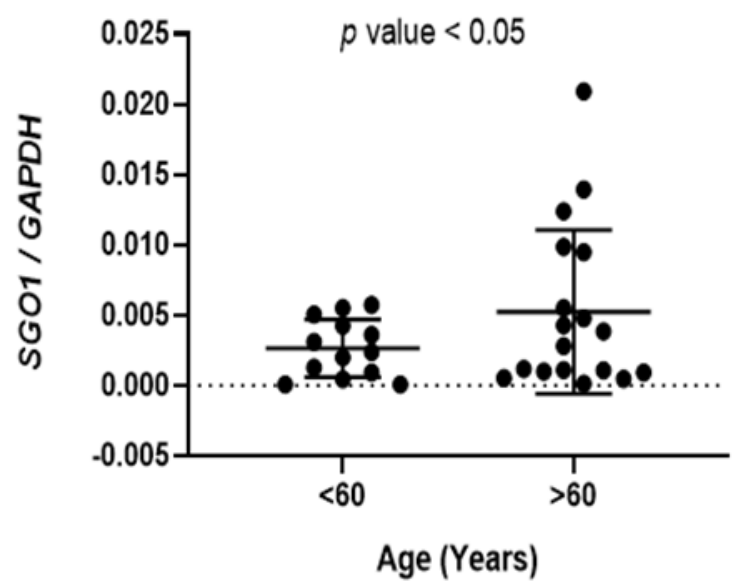

تصوير r. نمودار تغييرات سطح بيان SGO1-AS1 , SGO1 در كروه كمتر از •و سال و بيشتر از •وسال در بافتهاى تومورى

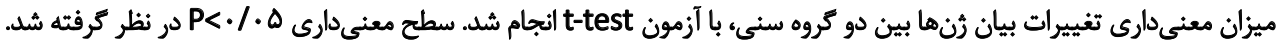

A

ROC curve: ROC of SGO1-AS1

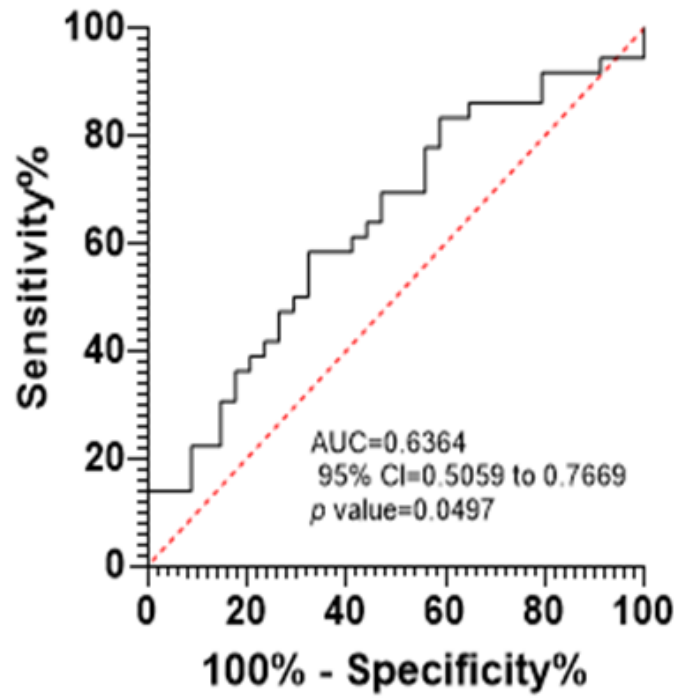

B

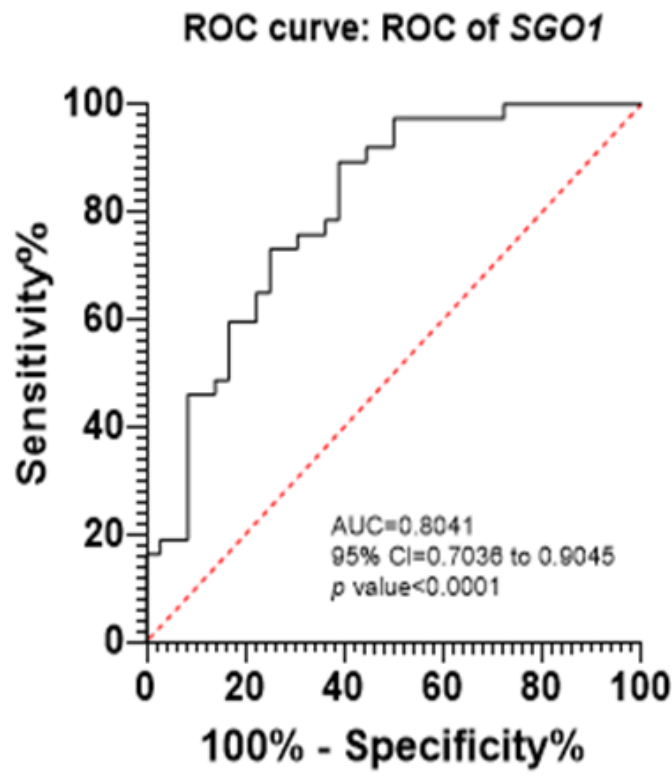




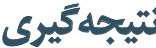

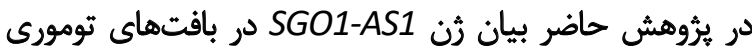

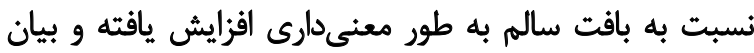

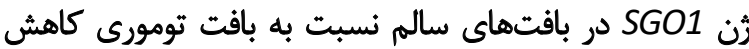

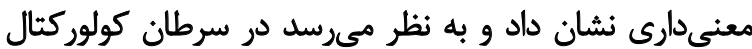

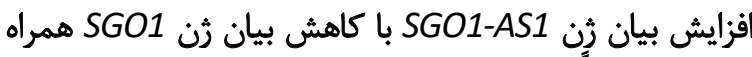

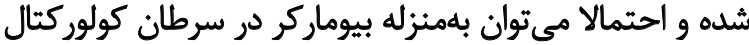

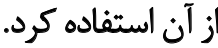

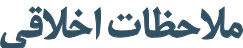

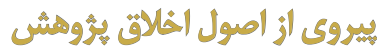

اين يُروهش در كميته اخلاق دانشكاه آزاد شهركرد با كد ابه تصويب رسيده است. R.IAU.SHKREC.1398.020

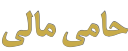

اين مقاله بركرفته بايايانامه كارشناسى ارشد نويسنده اول

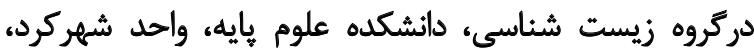

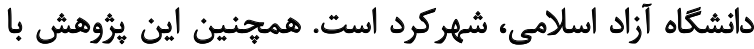

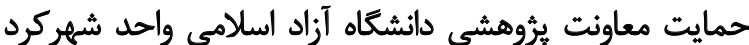

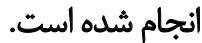

\section{مشاركت نويسند كان ون}

هر دو نويسندكان معيارهاى استاندارد نويسندكى بر اساس

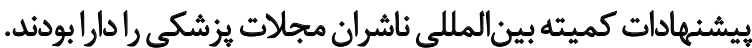

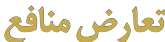

بنابر اظهار نويسندكان اين مقاله هيجكونه تعارض منافع وجود

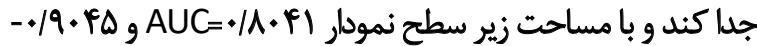

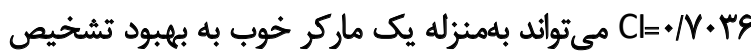
بيمارى سرطان كلوركتال كمك كند (تصوير شماره بان ب).

بحث

تا به امروز نرخ زيادى از مركىومير در سرطان كلوركتال كزارش

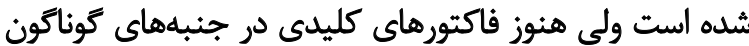

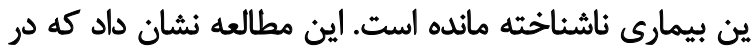

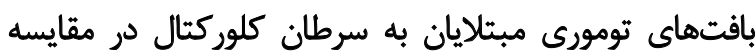

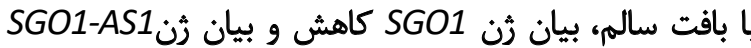

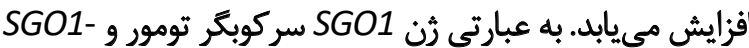

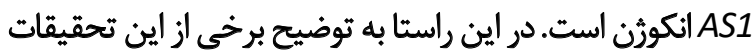

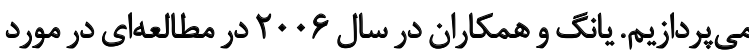

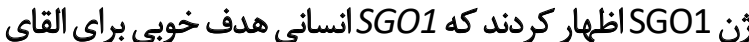

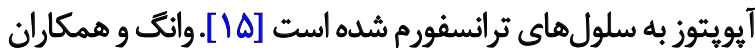

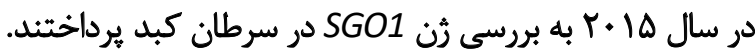

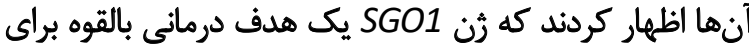

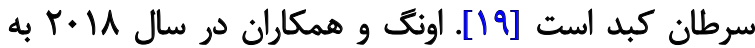

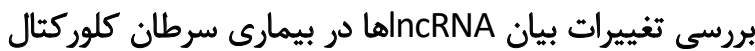

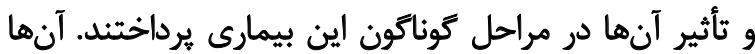

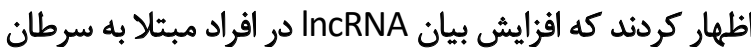

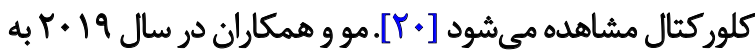

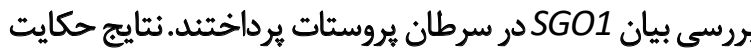

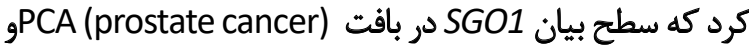

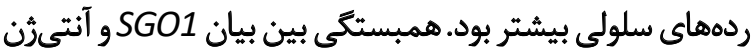

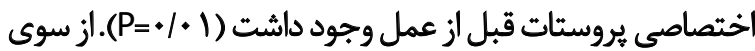

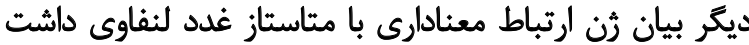

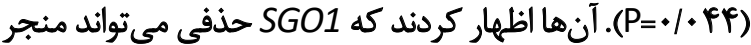

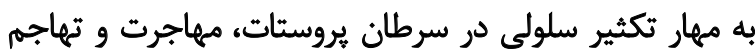

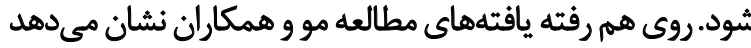

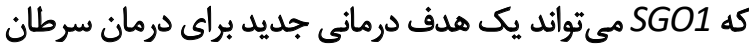

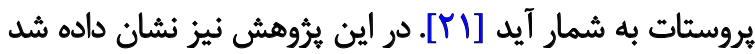

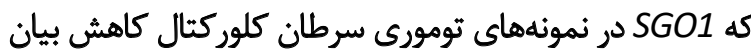

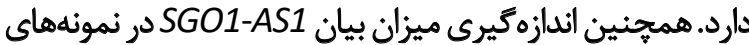

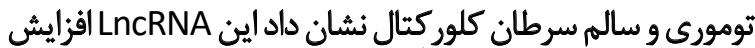

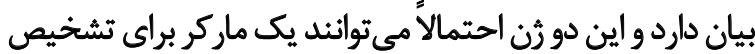
سرطان كلوركتال شناخته شوند.

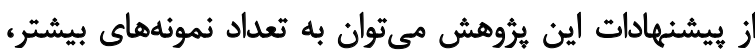

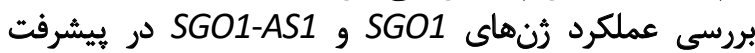

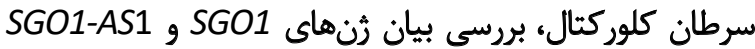

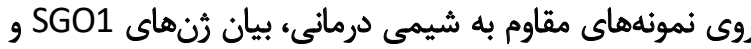
SGO1-AS1

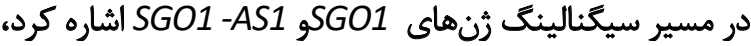

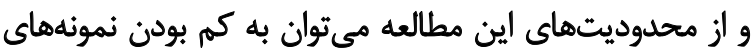
جمع آورى شده اشاره نمود. 


\section{Refrences}

[1] Geboes K, Ectors N, Geboes KP. Pathology of early lower Gl cancer. Best Pract Res Clin Gastroenterol. 2005; 19(6):963-78. [DOI:10.1016/j.bpg.2005.04.005][PMID]

[2] Ansari R, Mahdavinia M, Sadjadi A, Nouraie M, Kamangar F, Bishehsari $F$, et al. Incidence and age distribution of colorectal cancer in Iran: results of a population-based cancer registry. Cancer Lett. 2006; 240(1):143-7. [DOI:10.1016/j.canlet.2005.09.004][PMID]

[3] Burt RW, Barthel JS, Dunn KB, David DS, Drelichman E, Ford JM, et al. Colorectal cancer screening. J Natl Compr Canc Ne. 2010; 8(1):8-61. [DOI:10.6004/jnccn.2010.0003]

[4] Kumar V, Abbas AK, Aster JC. Robbins basic pathology e-book. Amsterdam: Elsevier Health Sciences; 2017. https://books.google.com/ books?id=YYZMDgAAQBAJ\&dq

[5] Pahlavan PS, Kanthan R. The epidemiology and clinical findings of colorectal cancer in Iran. J Gastrointestin Liver Dis. 2006; 15(1):15-9. [PMID]

[6] Mahapatra K, Roy S. An insight into the folding and stability of Arabidopsis thaliana SOG1 transcription factor under salinity stress in vitro. Biochem Biophys Res Commun. 2019; 515(4):531-7. [DOI:10.1016/j.bbrc.2019.05.183] [PMID]

[7] Piché J, Gosset N, Legault LM, Pacis A, Oneglia A, Caron M, et al. Molecular signature of CAID syndrome: Noncanonical roles of SGO1 in regulation of TGF- $\beta$ signaling and epigenomics. Cell Mol Gastroenterol Hepatol. 2019; 7(2):411-31. [DOI:10.1016/j.jcmgh.2018.10.011] [PMID]

[8] Mishra PK, Thapa KS, Chen P, Wang S, Hazbun TR, Basrai MA. Budding yeast CENP-ACse4 interacts with the N-terminus of SGO1 and regulates its association with centromeric chromatin. Cell Cycle. 2018; 17(1):11-23. [DOI:10.1080/15384101.2017.1380129] [PMID]

[9] Rao CV, Sanghera S, Zhang Y, Biddick L, Reddy A, Lightfoot S, et al. Systemic chromosome instability resulted in colonic transcriptomic changes in metabolic, proliferation, and stem cell regulators in SGO1-/+ Mice. Cancer Res. 2016; 76(3):630-42. [DOI:10.1158/00085472.CAN-15-0940]

[10] Ulitsky I, Bartel DP. lincRNAs: Genomics, evolution, and mechanisms. Cell. 2013; 154(1):26-46. [DOI:10.1016/j.cell.2013.06.020] [PMID]

[11] Kugel JF, Goodrich JA. Non-coding RNAs: Key regulators of mammalian transcription. Trends Biochem Sci. 2012; 37(4):144-51. [DOI:10.1016/j.tibs.2011.12.003] [PMID] [PMCID]

[12] Yao Y, Dai W. Shugoshins function as a guardian for chromosomal stability in nuclear division. Cell Cycle. 11(14):2631-42. [DOI:10.4161/ cc.20633] [PMID] [PMCID]

[13] Gooding AJ, Zhang B, Jahanbani FK, Gilmore HL, Chang JC, Valadkhan $\mathrm{S}$, et al. The IncRNA BORG drives breast cancer metastasis and disease recurrence. Sci Rep. 2017; 7(1):1-18. [DOI:10.1038/s41598017-12716-6]

[14] Nasim N, Ghafouri-Fard S, Soleimani S, Esfandi F, Shirkhoda M, Safaei M, et al. Assessment of SGO1 and SGO1-AS1 contribution in breast cancer. Hum Antibodies. 2019; 27(4):279-84.[DOI:10.3233/ HAB-190384] [PMID]

[15] Yang Y, Wang X, Dai W. Human SGO1 is an excellent target for induction of apoptosis of transformed cells. Cell Cycle. 2006; 5(8):896-901. [DOI:10.4161/cc.5.8.2691] [PMID]
[16] Thornton B, Basu C. Rapid and simple method of qPCR primer design. In: Basu C, editor. PCR Primer Design. Berlin; Springer; 2015. p. 173-9. [DOI:10.1007/978-1-4939-2365-6_13]

[17] Rychlik W. OLIGO 7 primer analysis software. In: Yuryev A, editor. PCR Primer Design. Berlin: Springer; 2007. p. 35-59. [DOI:10.1007/978-1-59745-528-2_2]

[18] Chen C, Tan R, Wong L, Fekete R, Halsey J. Quantitation of microRNAs by real-time RT-qPCR. In: Park DJ, PCR Protocols. Berlin: Springer; 2011. pp. 113-34. [DOI:10.1007/978-1-60761-944-4_8]

[19] Wang LH, Yen CJ, Li TN, Elowe S, Wang WC, Wang LHC. SGO1 is a potential therapeutic target for hepatocellular carcinoma. Oncotarget. 2015; 6(4):2023-33. [DOI:10.18632/oncotarget.2764][PMID] [PMCID]

[20] Ong MS, Cai W, Tan TZ, Huang RY-J, Hooi SC, Yap CT, et al. Long noncoding RNA landscape in colorectal cancer. RNA Dis. 2019; 6(2019):19. [DOI:10.14800/rd.1628]

[21] Mu J, Fan L, Liu D, Zhu D. Overexpression of shugoshin1 predicts a poor prognosis for prostate cancer and promotes metastasis by af fecting epithelial-mesenchymal transition. Onco Targets Ther. 2019; 12:1111-8. [DOI:10.2147/OTT.S191157] [PMID][PMCID] 